\title{
Increased mortality in patients with severe traumatic brain injury treated without intracranial pressure monitoring
}

\author{
Clinical article
}

\author{
Arash Farahvar, M.D., Ph.D., ${ }^{1}$ Linda M. Gerber, Ph.D., ${ }^{2}$ Ya-Lin Chiu, M.S., ${ }^{2}$ \\ Nancy Carney, Ph.D., ${ }^{3}$ Roger Härtl, M.D., ${ }^{4}$ and Jamshid Ghajar, M.D., Ph.D. ${ }^{4,5}$ \\ ${ }^{1}$ Department of Neurosurgery, University of Rochester Medical Center, Rochester; Departments of ${ }^{2}$ Public \\ Health and ${ }^{4}$ Neurological Surgery, Weill Cornell Medical College; and ${ }^{5}$ Brain Trauma Foundation, \\ New York, New York; and ${ }^{3}$ Department of Medical Informatics and Clinical Epidemiology, Oregon \\ Health \& Science University, Portland, Oregon
}

\begin{abstract}
Object. Evidence-based guidelines recommend intracranial pressure (ICP) monitoring for patients with severe traumatic brain injury (TBI), but there is limited evidence that monitoring and treating intracranial hypertension reduces mortality. This study uses a large, prospectively collected database to examine the effect on 2-week mortality of ICP reduction therapies administered to patients with severe TBI treated either with or without an ICP monitor.

Methods. From a population of 2134 patients with severe TBI (Glasgow Coma Scale [GCS] Score < 9), 1446 patients were treated with ICP-lowering therapies. Of those, 1202 had an ICP monitor inserted and 244 were treated without monitoring. Patients were admitted to one of 20 Level I and two Level II trauma centers, part of a New York State quality improvement program administered by the Brain Trauma Foundation between 2000 and 2009. This database also contains information on known independent early prognostic indicators of mortality, including age, admission GCS score, pupillary status, CT scanning findings, and hypotension.

Results. Age, initial GCS score, hypotension, and CT scan findings were associated with 2-week mortality. In addition, patients of all ages treated with an ICP monitor in place had lower mortality at 2 weeks $(p=0.02)$ than those treated without an ICP monitor, after adjusting for parameters that independently affect mortality.

Conclusions. In patients with severe TBI treated for intracranial hypertension, the use of an ICP monitor is associated with significantly lower mortality when compared with patients treated without an ICP monitor. Based on these findings, the authors conclude that ICP-directed therapy in patients with severe TBI should be guided by ICP monitoring.
\end{abstract}

(http://thejns.org/doi/abs/10.3171/2012.7.JNS111816)

\section{KEY WORDS • intracranial pressure • mortality rate • traumatic brain injury $\quad$ evidence-based guidelines}

$\mathrm{T}$ RAUMATIC brain injury remains a leading cause of death and disability in young people. In the US, an estimated 1.5 million people sustain TBI, resulting in over 50,000 deaths and 500,000 individuals with permanent neurological sequelae. This epidemic places an economic burden on the health care system, with annual costs estimated at over $\$ 64$ billion. ${ }^{18}$

The monitoring of ICP in patients with severe TBI is recommended in the BTF Guidelines for the Management of Severe TBI (referred to in this paper as the "Guidelines") and endorsed by the AANS, the CNS, and the Joint Neurotrauma and Critical Care section of the AANS/CNS. ${ }^{5}$

The significance of intracranial hypertension in pre-

Abbreviations used in this paper: AANS = American Association of Neurological Surgeons; BTF = Brain Trauma Foundation; CNS = Congress of Neurological Surgeons; GCS = Glasgow Coma Scale; $\mathrm{ICP}=$ intracranial pressure; $\mathrm{TBI}=$ traumatic brain injury. dicting outcome from TBI has been well studied since the 1960 s. $^{2,4,12,17,19,20}$ Aside from its prognostic value, ICP monitoring also guides early diagnosis and management of intracranial hypertension. ${ }^{29}$ Furthermore, there are now several evidence-based studies showing that ICP monitoring, when used in a protocol-based manner in neurosurgical ICUs, leads to improved outcomes in adjusted mortality rates for patients with severe TBI. A meta-analysis of clinical studies since 1970 found that patients who were aggressively monitored for ICP after severe TBI had a $12 \%$ lower mortality rate and $6 \%$ more favorable outcomes when compared with less intensely monitored patients. ${ }^{26}$

Despite growing adherence to the Guidelines recommendations $\mathrm{s}^{16}$ and increasing evidence showing that implementation leads to improved outcomes and reduced

This article contains some figures that are displayed in color online but in black-and-white in the print edition. 
costs, ${ }^{11,15,22}$ there is still controversy as to the impact of ICP monitoring on outcomes. Recent studies assert that there is no conclusive evidence for a beneficial effect for the placement of ICP monitors in patients with blunt head injury. ${ }^{1,9}$ Others state that ventriculostomy procedures are associated with both increased mortality rates and lower Functional Independence Measure scores. ${ }^{24}$

There are reports questioning the efficacy of ICP monitoring per se..$^{1,924,28}$ Methodological weaknesses in these studies include the following: 1) single-center studies; 2) retrospectively collected data; 3 ) lack of prognostic parameters for adjusted mortality rates; 4) exclusion of patients who died within the first 24 hours; 5) differences in treatment protocols; and 6) baseline differences between groups in severity as well as other disease characteristics known to affect outcome following TBI. ${ }^{27}$ Our study uses data collected prospectively from the BTF New York State database obtained from Level I and Level II trauma centers to analyze the effect of ICP monitoring on adjusted mortality rates among patients with severe TBI treated with ICP-lowering therapies. We hypothesized that patients with severe TBI who had an ICP monitor and were treated would have a lower mortality rate than patients who were treated without the use of ICP monitoring.

\section{Methods}

\section{The "TBI-Trac" Database}

As part of a quality improvement initiative for patients with severe TBI, the BTF designed and implemented a program that is funded by the New York State Department of Health, Division of Healthcare Financing and Acute and Primary Care Reimbursement. The program uses an online Internet database, TBI-trac, to collect prospective data on patients with severe TBI, to be used by trauma centers to track compliance with Guidelines recommendations and to test hypotheses that could have evidence-based impact on improving the Guidelines. This database contains clinical information about patients with severe TBI, information derived from prehospital sources, emergency department records, the first 10 days in the ICU, and 2-week mortality data from 22 trauma centers in New York State (20 Level I and 2 Level II). This report is based on patients treated in these trauma centers between June 6, 2000, and December 31, 2009.

The TBI-trac database contains all patients with isolated severe TBI or multiple trauma who meet the following criteria: arrival at the participating trauma center within 24 hours of injury; a GCS score $<9$, including a GCS motor score of $<6$ for at least 6 hours after injury and after resuscitation efforts (airway management, ventilatory support, and circulatory support); and mechanism of injury consistent with trauma.

The intervention group consisted of patients who received treatment and an ICP monitor, and the comparison group consisted of patients who received treatment without an ICP monitor. We used the following procedure to select cases from the TBI-trac database. Patients at risk for intracranial hypertension were selected for the analysis if they had an abnormal CT scan or at least 2 of the following 3 criteria: age $>40$ years, hypotension, or a GCS motor score of 1,2 , or $3 .{ }^{21}$ Of the group meeting these criteria, patients were further selected if at least one of the following ICP treatment regimens was administered in the first 2 days following admission: mannitol, hypertonic saline, barbiturates, drainage of CSF, or decompressive craniectomy. Patients were included in the analysis if any single treatment regimen or a combination of multiple therapeutic interventions aimed to reduce elevated ICP had been administered. Patients included in the monitored group had an ICP monitor inserted within the first 2 days of admission.

Patients with severe TBI who died in the emergency department or were admitted with the diagnosis of brain death were not included. Nonparalyzed patients on Day 1 or 2 following trauma, with a GCS score of 3 or 4, and with fixed and dilated pupils were recorded but excluded from data analysis, because these patients were unlikely to benefit from intervention. Those with no outcome data (37 patients) were excluded from the analysis.

Mortality was defined as 14-day postinjury assessment of the patient's condition as alive or dead. This time frame was selected because over $85 \%$ of deaths occur within 2 weeks after injury..$^{10}$ The research protocol was approved or deemed exempt from review by the institutional review boards of each of the participating centers. In compliance with Health Insurance Portability and Accountability Act regulations, the database refrains from the use of patient identifiers, thereby ensuring confidentiality for the data sets at each institution. All patient data and outcomes were recorded from the patient's medical records and entered directly into the TBI-trac database by a trained trauma nurse-coordinator at each of the participating centers. Quality assurance reviews of the data were performed and reports provided to each center. Quarterly reports to the centers included outlier data. The TBI-trac database includes automatic mechanisms for identification of out-of-range data.

\section{Statistical Analysis}

The chi-square test and t-tests were used where appropriate to compare characteristics between patients treated with and without the use of ICP monitoring. The means are expressed \pm SD. Multivariable logistic regression analyses, for the total sample and for adults (age $\geq 16$ years), were used to evaluate the association between ICP monitoring status and 2-week mortality, controlling for age, hypotension status on Day 1, pupillary status on Day 1 , initial GCS score, and CT scan results. The odds ratios, 95\% confidence intervals, and $\mathrm{p}$ values of the covariates were reported. All statistical tests were 2-sided, and $\mathrm{p}<$ 0.05 was considered statistically significant. Analyses were performed using SAS version 9.2 software (SAS Institute, Inc.).

\section{Results}

Characteristics of the Study Population by ICP Monitoring Status

Between June 6, 2000, and December 31, 2009, 3125 


\section{Death from severe TBI in patients treated without ICP monitoring}

patients were entered into the database. After application of the exclusion criteria, 2134 patients were eligible for the study. A total of 1446 patients of all ages were treated for intracranial hypertension. Patients were further subdivided into 2 groups; one with patients of all ages, including a pediatric population age $<16$ years (139 patients), and the other of adults only, age $\geq 16$ years (1307 patients). Table 1 shows the demographic and clinical characteristics of the study population. The mean age was $36.6 \pm 19.8$ years, over $75 \%$ of the patients were male, and $14.2 \%$ were $\geq 60$ years. Roughly equal numbers had a GCS score of 3-5 (55.7\%) and 6-8 (44.3\%). Hypotension on admission was found in $16 \%$ of the population. In adult patients (age $\geq$ 16 years), the mean age was $39.5 \pm 18.5$ years, with $77.1 \%$ male. Admission GCS score and percent hypotensive on admission were similar to the overall population (including all ages).

Of the 1307 adult patients who were treated on Day 1 and/or 2, the majority, $82.9 \%$ (1084 patients) had an ICP monitor placed (see Fig. 1). The mortality rate for those treated with an ICP monitor was $19.6 \%$ (212 patients), compared with $33.2 \%$ (74) in those treated without an ICP monitor. The characteristics of the adult study population by ICP monitoring status are shown in Table 2. Patients who were not monitored (223 patients) were significantly older $(\mathrm{p}<0.0001)$ and had more pupillary abnormalities than those who received monitoring ( $\mathrm{p}=$ 0.003 ). Patients who were not monitored did not differ from those monitored in terms of sex, initial GCS score, arterial hypotension, or CT abnormalities on Day 1.

\section{Factors Predicting 2-Week Mortality}

Multivariable logistic regression models predicting 2-week mortality for the total sample and for adults are shown in Table 3. In the total population (all ages; 1446 patients), ICP monitoring was a statistically significant predictor of 2-week mortality (OR $0.63,95 \%$ CI $0.41-0.94$; $\mathrm{p}=0.02$ ), after controlling for 5 covariates. Additional independent significant risk factors included older age, lower GCS score, CT abnormalities, and presence of hypotension on Day 1. These same risk factors held for the adult population. In the adult population a trend toward reduced risk was observed for patients having ICP monitoring (OR 0.64, 95\% CI 0.41-1.00; $\mathrm{p}=0.05$ ).

\section{Post Hoc Analysis}

We analyzed the distribution of ICP monitoring across centers to consider whether lack of monitoring was concentrated in a single or a few centers, and was thus skewing the results. Only 3 centers monitored less than $60 \%$ of eligible patients, and the number of patients monitored was 11 of 33 eligible patients, contributing $0.9 \%$ of the total monitored group (11 of 1202).

\section{Discussion}

\section{Use of ICP Monitoring and Its Outcome}

The findings of this study suggest that treatment of intracranial hypertension with the use of ICP monitoring, as recommended by the BTF Guidelines, improves
TABLE 1: Characteristics of study population treated for ICP elevation*

\begin{tabular}{lcc}
\hline \multicolumn{1}{c}{ Characteristic } & Adults (\%) & Total (\%) \\
\hline no. of pts & 1307 & 1446 \\
age (yrs) & & \\
$\quad$ mean \pm SD & $39.5 \pm 18.5$ & $36.6 \pm 19.8$ \\
$\quad$ range & $16-93$ & $0-93$ \\
age & & \\
$\quad<60$ & $1101(84.2)$ & $1240(85.8)$ \\
$\quad$ 60 & $206(15.8)$ & $206(14.2)$ \\
sex & & \\
$\quad$ male & $1007(77.1)$ & $1094(75.7)$ \\
$\quad$ female & $300(22.9)$ & $352(24.3)$ \\
initial GCS score & & \\
6-8 & $545(44.3)$ & $606(44.3)$ \\
3-5 & $685(55.7)$ & $761(55.7)$ \\
hypotension present on Day 1 & & \\
$\quad$ yes & $208(16.0)$ & $229(16.0)$ \\
no & $1094(84.0)$ & $1207(84.0)$ \\
CT scan findings & & \\
normal \\
abnormal \\
pupillary abnormalities \\
yes \\
no
\end{tabular}

* Adulthood was defined as age $\geq 16$ years. Abbreviation: pts = patients.

outcome as measured by 2 -week adjusted mortality. The characteristics of the patients in this study treated for intracranial hypertension with monitoring versus those treated without a monitor did not differ with regard to sex, initial GCS score, presence of systemic hypotension, or CT scan findings. However, 2-week mortality was significantly improved if treatment was coupled with the use of an ICP monitor. The use of initial Day 1 postresuscitation GCS scores, CT scan parameters, or presence of arterial hypotension may not delineate patients who will benefit from ICP monitoring and treatment. Similarly, in a previous study ${ }^{14}$ these same variables did not independently predict therapeutic response to the treatment of ICP elevation.

\section{Limitations of This Study}

There are 4 key limitations to this study.

Lack of Information About Patients Who Were Not Monitored. Data were not collected to indicate why a physician did not place an ICP monitor in a qualified patient at risk for intracranial hypertension. Nor does this study answer why the medical decision was made to treat the 
A. Farahvar et al.

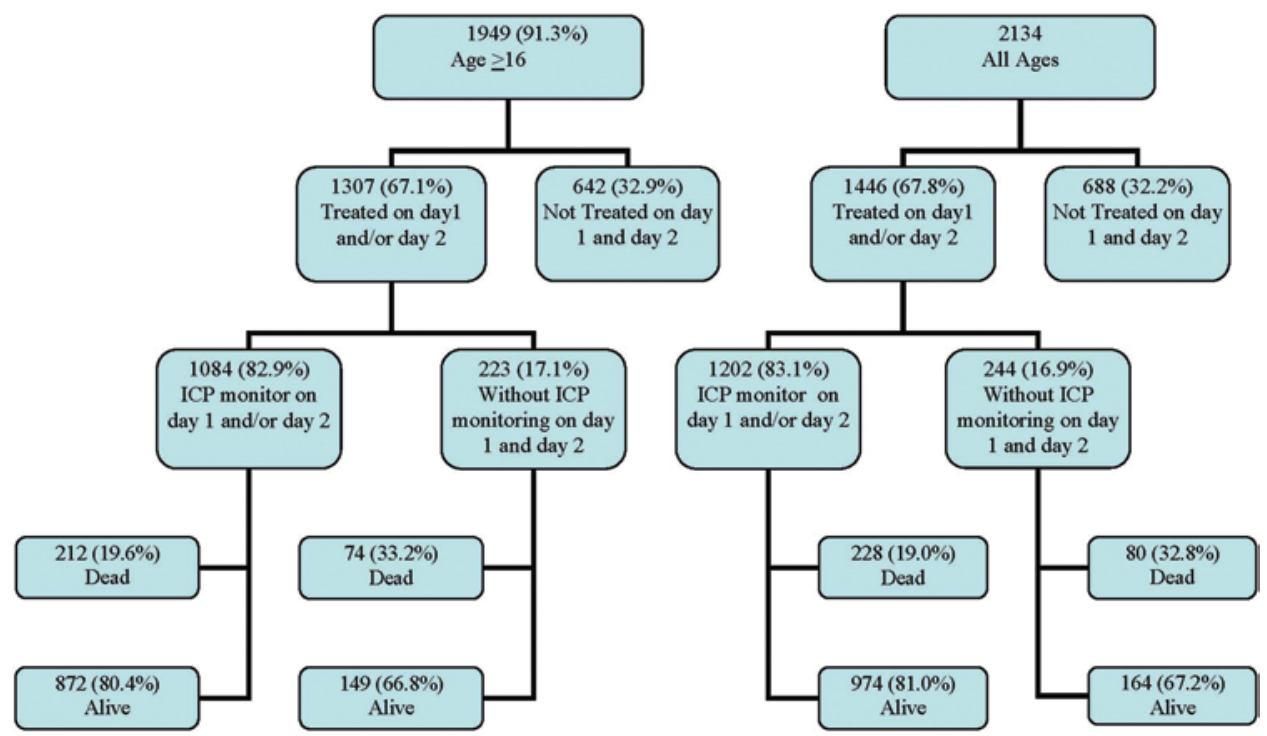

FIG. 1. Flowchart showing the process of identifying those patients who received treatment and monitoring on Days 1 and 2.

TABLE 2: Characteristics of adult study population treated for ICP elevation, according to ICP monitoring status

\begin{tabular}{|c|c|c|c|}
\hline Characteristic & $\begin{array}{l}\text { No ICP Monitor- } \\
\text { ing (\%) }\end{array}$ & $\begin{array}{l}\text { ICP Monitor- } \\
\text { ing (\%) }\end{array}$ & $p$ Value \\
\hline no. of pts & 223 & 1084 & \\
\hline \multicolumn{4}{|l|}{ age (yrs) } \\
\hline mean $\pm S D$ & $45.9 \pm 21.8$ & $38.3 \pm 17.5$ & $<0.0001$ \\
\hline range & $16-89$ & $16-93$ & \\
\hline \multicolumn{4}{|l|}{ age } \\
\hline$<60$ & $158(70.9)$ & $943(87.0)$ & $<0.0001$ \\
\hline$\geq 60$ & $65(29.1)$ & $141(13.0)$ & \\
\hline \multicolumn{4}{|l|}{ sex } \\
\hline male & $164(73.5)$ & $843(77.8)$ & 0.17 \\
\hline female & $59(26.5)$ & $241(22.2)$ & \\
\hline \multicolumn{4}{|l|}{ initial GCS score } \\
\hline $6-8$ & $96(48.5)$ & $449(43.5)$ & 0.20 \\
\hline $3-5$ & $102(51.5)$ & $583(56.5)$ & \\
\hline \multicolumn{4}{|c|}{$\begin{array}{l}\text { hypotension present on } \\
\text { Day } 1\end{array}$} \\
\hline yes & $38(17.0)$ & $170(15.8)$ & 0.63 \\
\hline no & $185(83.0)$ & 909 (84.2) & \\
\hline \multicolumn{4}{|l|}{ CT scan findings } \\
\hline normal & $9(4.1)$ & $35(3.3)$ & 0.52 \\
\hline abnormal & $209(95.9)$ & $1037(96.7)$ & \\
\hline \multicolumn{4}{|c|}{ pupillary abnormalities } \\
\hline yes & $72(32.6)$ & $245(23.0)$ & 0.003 \\
\hline no & $149(67.4)$ & $821(87.0)$ & \\
\hline \multicolumn{4}{|l|}{ 2-wk outcome } \\
\hline alive & $149(66.8)$ & $872(80.4)$ & $<0.0001$ \\
\hline dead & $74(33.2)$ & $212(19.6)$ & \\
\hline
\end{tabular}

patient without an ICP monitor in place. It could be that physicians decided not to monitor in those patients whose condition was considered more severe and potentially not salvageable. However, if that were the case the question remains: why were those patients treated? Learning the rationale for decisions to monitor or not, and to treat or not, is a topic requiring investigation, and beyond the scope of this project. In our analysis we controlled for variables often associated with greater injury severity, and lack of monitoring remained a significant predictor of mortality.

Short-Term Outcomes. Two-week adjusted mortality rather than 6-month Glasgow Outcome Scale score was used as the primary outcome measure. Two-week mortality was chosen as the end point of this study because this early time point accounts for over $85 \%$ of all TBI-related mortality and more appropriately reflects the severity of the injury as well as the efficacy of early intervention, whereas later time points such as 30-day mortality include complications or associated comorbidities due to ICU and hospital length of stay. ${ }^{23}$ A future goal of our program is to extend our outcome assessment to include long-term functional status, to better reflect the influence of early interventions on patients and families.

Study Design. A well-controlled, randomized clinical trial comparing outcomes for patients who are monitored with those who are not, with both groups being treated for intracranial hypertension, would answer our research question with more confidence than the current, prospective design. Whereas such a study is unlikely to occur in the US, there is one being conducted in Latin America (where ICP monitoring is not the standard of care in most areas) that may further elucidate the role of ICP monitoring in patients with severe TBI (N. Carney, personal communication, 2011).

Specific Treatments and Outcome. This study did not identify specific therapeutic strategies initiated after the 


\section{Death from severe TBI in patients treated without ICP monitoring}

TABLE 3: Logistic regression analyses predicting 2-week mortality for all 1446 patients and for the subpopulation of 1307 adult patients

\begin{tabular}{|c|c|c|c|c|}
\hline \multirow[b]{2}{*}{ Predictor Variable } & \multicolumn{2}{|l|}{ Adults } & \multicolumn{2}{|l|}{ All Ages } \\
\hline & Adjusted OR (95\% Cl) & p Value & Adjusted OR (95\% Cl) & $p$ Value \\
\hline \multicolumn{5}{|l|}{ ICP monitoring } \\
\hline yes & $0.64(0.41-1.00)$ & 0.05 & $0.63(0.41-0.94)$ & 0.02 \\
\hline no & reference & & reference & \\
\hline \multicolumn{5}{|l|}{ age (yrs) } \\
\hline$\geq 60$ & $2.43(1.56-3.79)$ & $<0.0001$ & $2.50(1.65-3.78)$ & $<0.0001$ \\
\hline$<60$ & reference & & reference & \\
\hline \multicolumn{5}{|l|}{ initial GCS score } \\
\hline $6-8$ & $0.46(0.37-0.57)$ & $<0.0001$ & $0.44(0.36-0.53)$ & $<0.0001$ \\
\hline $3-5$ & reference & & reference & \\
\hline \multicolumn{5}{|c|}{ hypotension present on Day 1} \\
\hline yes & $2.08(1.48-2.92)$ & $<0.0001$ & $2.08(1.54-2.82)$ & $<0.0001$ \\
\hline no & reference & & reference & \\
\hline \multicolumn{5}{|l|}{ CT scan findings } \\
\hline abnormal & $2.45(1.05-5.75)$ & 0.04 & $2.71(1.11-6.60)$ & 0.03 \\
\hline normal & reference & & reference & \\
\hline \multicolumn{5}{|c|}{ pupil abnormalities on Day 1} \\
\hline yes & $1.38(0.99-1.91)$ & 0.05 & $1.40(0.98-2.00)$ & 0.07 \\
\hline no & reference & & reference & \\
\hline
\end{tabular}

placement of an ICP monitor that may affect survival. Similarly, there may be additional physiological variables that were not measured that may affect the decision to place an ICP monitor and affect subsequent survival rates.

\section{Factors Influencing the Decision to Monitor}

The relationship between age and worse outcome, with a significantly higher mortality rate, has been reported before..$^{3,6,7,13}$ Sorrentino et al..$^{25}$ found differences in cerebral autoregulation and cerebral pressure reactivity index in elderly patients with TBI. However, there is also evidence that older patients may be more responsive to intracranial hypertension treatment. ${ }^{14}$

In this study patients with pupillary abnormalities were less likely to be monitored. Recent evidence may contradict the idea that patients with a GCS score of 3 with anisocoria have universally poor outcomes or are unsalvageable. Chamoun et al. ${ }^{8}$ showed that patients with a GCS score of 3 had an overall survival rate of $50.8 \%$. They also found that $25.5 \%$ of patients with bilateral reactive pupils and $27.6 \%$ with unilateral fixed and dilated pupils had a good outcome (Glasgow Outcome Scale score of 1 or 2) at 6 months.

\section{Conclusions}

Despite evidence-based guidelines that support the role of ICP monitoring in the management of severe TBI, there still exist significant variations in practice, and patients undergo ICP-lowering therapy without an ICP monitor. The database used in this study contains prospectively collected information on a large number of patients with severe TBI. Previous studies on the use of
ICP monitoring have largely relied on meta-analyses of smaller retrospective studies, or larger databases that did not include parameters needed to adjust for the risk of mortality and to address the independent effect of ICP monitoring. The findings of this prospective study suggest that ICP monitoring in conjunction with treatment is associated with a significantly reduced risk of death compared with treatment without an ICP monitor. Future research must include analysis of the rationale for clinicians' decisions to monitor or not, to understand more fully the relationship between ICP monitor-based treatment and outcome.

\section{Disclosure}

The authors thank the New York State Department of Health and the New York Presbyterian Hospital TBI fund for their financial support. Dr. Härtl is a consultant for Brainlab, Synthes, and SpineWave. Dr. Ghajar is president of the Brain Trauma Foundation. The authors report no conflict of interest concerning the materials or methods used in this study or the findings specified in this paper.

Author contributions to the study and manuscript preparation include the following. Conception and design: Ghajar, Gerber, Härtl. Acquisition of data: Gerber, Chiu. Analysis and interpretation of data: Farahvar, Gerber, Chiu, Carney, Härtl. Drafting the article: Ghajar, Farahvar, Gerber, Chiu, Härtl. Critically revising the article: all authors. Reviewed submitted version of manuscript: all authors. Approved the final version of the manuscript on behalf of all authors: Ghajar. Administrative/technical/material support: Gerber. Study supervision: Ghajar.

\section{References}

1. Akopian G, Gaspard DJ, Alexander M: Outcomes of blunt head trauma without intracranial pressure monitoring. Am Surg 73:447-450, 2007 
2. Alberico AM, Ward JD, Choi SC, Marmarou A, Young HF: Outcome after severe head injury. Relationship to mass lesions, diffuse injury, and ICP course in pediatric and adult patients. J Neurosurg 67:648-656, 1987

3. Bhullar IS, Roberts EE, Brown L, Lipei H: The effect of age on blunt traumatic brain-injured patients. Am Surg 76:966968, 2010

4. Born JD, Hans P, Bonnal J: [Intracranial pressure in severe brain injuries. 2nd Part: Therapeutic interests and prognosis.] Neurochirurgie 30:329-334, 1984 (Fr)

5. Bratton SL, Chestnut RM, Ghajar J, McConnell Hammond FF, Harris OA, Hartl R, et al: Guidelines for the management of severe traumatic brain injury. VI. Indications for intracranial pressure monitoring. J Neurotrauma 24 (Suppl 1):S37-S44, 2007 (Erratum in J Neurotrauma 25:276-278, 2008)

6. Brazinova A, Mauritz W, Leitgeb J, Wilbacher I, Majdan M, Janciak I, et al: Outcomes of patients with severe traumatic brain injury who have Glasgow Coma Scale scores of 3 or 4 and are over 65 years old. J Neurotrauma 27:1549-1555, 2010

7. Bullock R, Chesnut RM, Clifton G, Ghajar J, Marion DW, Narayan RK, et al: Management and Prognosis of Severe Traumatic Brain Injury, ed 3. New York: Brain Trauma Foundation, 2000

8. Chamoun RB, Robertson CS, Gopinath SP: Outcome in patients with blunt head trauma and a Glasgow Coma Scale score of 3 at presentation. Clinical article. J Neurosurg 111: 683-687, 2009

9. Cremer OL, van Dijk GW, van Wensen E, Brekelmans GJ, Moons KG, Leenen LP, et al: Effect of intracranial pressure monitoring and targeted intensive care on functional outcome after severe head injury. Crit Care Med 33:2207-2213, 2005

10. Edwards WH: Patient safety in the neonatal intensive care unit. Clin Perinatol 32:97-106, vi, 2005

11. Fakhry SM, Trask AL, Waller MA, Watts DD: Management of brain-injured patients by an evidence-based medicine protocol improves outcomes and decreases hospital charges. J Trauma 56:492-500, 2004

12. Falcão AL, Dantas Filho VP, Sardinha LA, Quagliato EM, Dragosavac D, Araújo S, et al: Highlighting intracranial pressure monitoring in patients with severe acute brain trauma. Arq Neuropsiquiatr 53 (3-A):390-394, 1995

13. Falk AC: Age differences in brain injury characteristics. Dev Neurorehabil 13:315-321, 2010

14. Farahvar A, Gerber LM, Chiu YL, Härtl R, Froelich M, Carney N, et al: Response to intracranial hypertension treatment as a predictor of death in patients with severe traumatic brain injury. Clinical article. J Neurosurg 114:1471-1478, 2011 (Erratum in J Neurosurg 115:191, 2011)

15. Faul M, Wald MM, Rutland-Brown W, Sullivent EE, Sattin RW: Using a cost-benefit analysis to estimate outcomes of a clinical treatment guideline: testing the Brain Trauma Foundation guidelines for the treatment of severe traumatic brain injury. J Trauma 63:1271-1278, 2007

16. Hesdorffer DC, Ghajar J: Marked improvement in adherence to traumatic brain injury guidelines in United States trauma centers. J Trauma 63:841-848, 2007
17. Kasoff SS, Lansen TA, Holder D, Filippo JS: Aggressive physiologic monitoring of pediatric head trauma patients with elevated intracranial pressure. Pediatr Neurosci 14:241-249, 1988

18. Langlois JA, Rutland-Brown W, Thomas KE: Traumatic Brain Injury in the United States: Emergency Department Visits, Hospitalizations, and Deaths. Atlanta: Centers for Disease Control and Prevention, National Center of Injury Prevention and Control, 2004

19. Lundberg N: Continuous recording and control of ventricular fluid pressure in neurosurgical practice. Acta Psychiatr Scand Suppl 36:1-193, 1960

20. Miller JD, Becker DP, Ward JD, Sullivan HG, Adams WE, Rosner MJ: Significance of intracranial hypertension in severe head injury. J Neurosurg 47:503-516, 1977

21. Narayan RK, Michel ME, Ansell B, Baethmann A, Biegon A, Bracken MB, et al: Clinical trials in head injury. J Neurotrauma 19:503-557, 2002

22. Palmer S, Bader MK, Qureshi A, Palmer J, Shaver T, Borzatta $\mathrm{M}$, et al: The impact on outcomes in a community hospital setting of using the AANS traumatic brain injury guidelines. J Trauma 50:657-664, 2001

23. Roberts SR, Hamedani B: Benefits and methods of achieving strict glycemic control in the ICU. Crit Care Nurs Clin North Am 16:537-545, 2004

24. Shafi S, Diaz-Arrastia R, Madden C, Gentilello L: Intracranial pressure monitoring in brain-injured patients is associated with worsening of survival. J Trauma 64:335-340, 2008

25. Sorrentino E, Diedler J, Kasprowicz M, Budohoski KP, Haubrich C, Smielewski P, et al: Critical thresholds for cerebrovascular reactivity after traumatic brain injury. Neurocrit Care 16:258-266, 2012

26. Stein SC, Georgoff P, Meghan S, Mirza KL, El Falaky OM: Relationship of aggressive monitoring and treatment to improved outcomes in severe traumatic brain injury. Clinical article. J Neurosurg 112:1105-1112, 2010

27. Stover JF, Steiger P, Stocker R: Need for intracranial pressure monitoring following severe traumatic brain injury. Crit Care Med 34:1582-1584, 2006 (Letter)

28. Stuart GG, Merry GS, Smith JA, Yelland JD: Severe head injury managed without intracranial pressure monitoring. J Neurosurg 59:601-605, 1983

29. Unterberg A, Kiening K, Schmiedek P, Lanksch W: Longterm observations of intracranial pressure after severe head injury. The phenomenon of secondary rise of intracranial pressure. Neurosurgery 32:17-24, 1993

Manuscript submitted October 13, 2011

Accepted July 11, 2012.

Please include this information when citing this paper: published online August 17, 2012; DOI: 10.3171/2012.7.JNS111816.

Address correspondence to: Jamshid Ghajar, M.D., Ph.D., Brain Trauma Foundation, 7 World Trade Center, 34th Floor, 250 Greenwich Street, New York, New York 10007. email: ghajar@ braintrauma.org. 